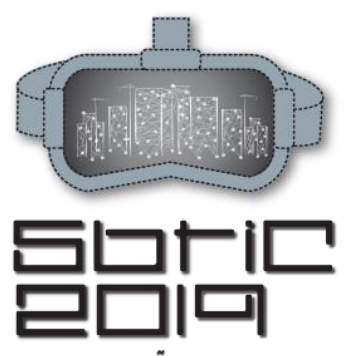

VIRTUALIZAÇÃם INTELIGENTE NQ PRDJETQ E NA CONSTRUÇÃa $2^{\circ}$ Simpósio Brasileiro de Tecnologia da Informação e Comunição na Construção

UNICAMP | 19 a 21 de agosto
INTEGRAÇÃO ENTRE AS TECNOLOGIAS BIM E
BLOCKCHAIN PARA O CONTROLE DO REGISTRO
AUTORAL NA ÁREA DA CONSTRUÇÃO CIVIL
Integration between the BIM and Blockchain
Technologies for Authorship Control in the AEC Industry

Duílio Helfenstens Penques da Silva

Instituto Federal do Tocantins | Palmas, TO | duiliohelf@gmail.com

Elen Oliveira Vianna

Instituto Federal do Tocantins | Palmas, TO | elen@ifto.edu.br

Gilson Marafiga Pedroso

Instituto Federal do Tocantins | Palmas, TO | gilson@ifto.edu.br

\begin{abstract}
RESUMO
Este artigo descreve uma proposta para inserir a tecnologia Blockchain no fluxo de trabalho da tecnologia BIM. Em contraste com a metodologia de desenvolvimento de projetos de última geração, a tecnologia BIM incentiva os profissionais a trocar informações em todas as etapas de um projeto. À medida que a adoção da tecnologia BIM evolui, preocupações relacionadas à confiança, autoria e responsabilidade começam a surgir. A tecnologia Blockchain permite que informações importantes sejam registradas sem a possibilidade de modificações. Esta pesquisa investigou a potencial integração entre as duas tecnologias (BIM e Blockchain) a partir da simulação de um fluxo de trabalho em tecnologia BIM com o uso de uma ferramenta notarial de código aberto baseada no protocolo Blockchain Ethereum. A ferramenta adotada foi testada e seu potencial custo operacional foi analisado. Na última etapa, foi apresentado um fluxo de trabalho BIM aprimorado mostrando como Blockchain pode ser usado. Os resultados deste estudo mostraram que o software Blockchain escolhido pode ser usado na tecnologia BIM, e que a alta freqüência de troca de informações em BIM pode afetar os custos operacionais. O valor desta pesquisa é divulgar uma solução relevante para a problemática de confiança, autoria e responsabilidade dentro da tecnologia BIM.
\end{abstract}

Palavras-chave BIM; Blockchain; Ethereum; Notarização.

\begin{abstract}
This paper presents a proposal for integrating Blockchain technology within the Building Information Modeling (BIM) technology workflow. In contrast to the last generation's projects development methodology, BIM technology strongly encourages professionals to exchange information in all stages of a project. As BIM technology adoption evolves, issues regarding trust, authorship and accountability begin to arise. Blockchain technology allows important information to be registered and not allowing further modifications. This research investigated the potential integration between the two technologies by first selecting and simulating a BIM workflow, second, choosing an open source notary tool based on Ethereum Blockchain protocol. The adopted tool was tested and its potential operational cost was taken in consideration. In the last step, an improved BIM workflow was presented showing how Blockchain can be used. This study results showed that the chosen Blockchain software may be used in BIM technology, and that the high frequency of information exchange in BIM might affect operational costs. The core value of this research is to present a potential solution to issues concerning trust, authorship and accountability within BIM technology.
\end{abstract}

Keywords BIM: Blockchain; Ethereum; Notarization.

\title{
1 INTRODUÇÃO
}

A Building Information Modeling (BIM) é uma tecnologia empregada para concepção, projeto, execução e manutenção de empreendimentos da construção civil. Esta tecnologia tem por premissa a ampla troca de informações entre os entes envolvidos (ex.: arquitetos, engenheiros, construtores) e, embora ainda tenha sido empregada de modo limitado em determinados níveis e etapas, o emprego da mesma é desejável desde as etapas iniciais até as etapas finais do ciclo de vida de um empreendimento. Assim, em um nível ideal de adoção, cria-se um modelo BIM único de dados de projeto comum a todos os entes. Com base no referencial teórico, identificamos que o avanço na construção e o emprego do modelo BIM podem implicar em dificuldades relacionadas a direitos autorais e responsabilização de autores.

De acordo com Sacks et al. (2018, p. 14), a tecnologia BIM desempenha o papel de uma plataforma de suporte para o ciclo de vida de um empreendimento, pois é uma fonte de informações que permite a consulta,

SILVA, D. H. P.; VIANNA, E. O.; PEDROSO, G. M. Integração entre as tecnologias BIM e Blockchain para o controle do registro autoral na área da construção civil. In: SIMPÓSIO BRASILEIRO DE TECNOLOGIA DA INFORMAÇÃO E COMUNICAÇÃO NA CONSTRUÇÃO, 2., 2019, Campinas. Anais [...]. Porto Alegre: ANTAC, 2019. Disponível em: https://www.antaceventos.net.br/index.php/sbtic/sbtic2019/paper/view/216 
visualização e o reuso da informação corrente. Este ciclo é, portanto, altamente dependente das informações que constituem o modelo.

As metodologias de projeto precursoras ao emprego da tecnologia BIM operam de modo que as informações de um empreendimento sejam disponibilizadas à medida que cada projeto (arquitetônico, estrutural ou hidráulico, por exemplo) é disponibilizado. Entende-se, nesse sentido, que o autor de cada projeto é completamente responsável pelas informações contidas nele.

A adoção integral da tecnologia BIM passa pela superação de diversos desafios relacionados à "autoria dos dados" contidos nos modelos BIM. Para a CBIC, (2016a, p. 45), embora a questão da propriedade intelectual ainda não esteja resolvida, já levanta preocupações, uma vez que o emprego da metodologia BIM implica em um fluxo de informações "realizado de maneira mais livre". Segundo Foster (2008), determinadas peças contidas no modelo BIM não são necessariamente de autoria do projetista responsável. Para Schapke et al. (2018), a autoria pela informação pode ser transferida em função da modificação de elementos, ou até mesmo de atributos. Udom (2012) destaca o problema de atividades realizadas em conjunto, pois é difícil reconhecer aonde a contribuição de determinado ente inicia e termina. McAdam (2010), levanta, como exemplo, a possibilidade de a construtora contratada modificar o modelo BIM e suas implicações. Sacks et al. (2018, p. 358) apresentam questionamentos sobre quem é o proprietário das informações, quem tem os direitos legais sobre o modelo BIM como um todo, quem tem o direito de alterar o modelo e quem é responsável por eventuais problemas advindos de contribuições com informações imprecisas. Finalmente, para Stougiannos e Magneron (2018), uma preocupação quanto à adoção de BIM está relacionada à identidade de quem modifica o modelo BIM, pois há implicações sobre o autor durante e depois da execução da obra.

Os dispositivos da tecnologia da informação têm, ao longo dos anos, agregado valor à indústria da construção civil. Nos últimos anos, uma tecnologia nomeada como Blockchain tem servido como ferramenta para o registro imutável de informações a diversas indústrias. A imutabilidade dos registros em Blockchain tem permitido a estas indústrias solucionar problemáticas relacionadas à rastreabilidade de produtos, registro de propriedades e reclamação de autorias.

A tecnologia Blockchain é descrita por Zheng et al. (2017) como um registro público onde transações são armazenadas em blocos sequenciais. É natural desta tecnologia que cada bloco gerado contenha informações-chave do bloco anterior, formando assim uma cadeia de blocos. Ainda segundo Zheng et al. (2017) a segurança desta tecnologia é complementada pela distribuição dos dados registrados entre diversos usuários da "cadeia de blocos" e pelo uso de criptografia. As principais características da tecnologia Blockchain são descentralização, perenidade, anonimato e auditabilidade de dados.

Reconhecido o valor da tecnologia Blockchain e sua potencial sinergia com a tecnologia BIM este artigo tem como objetivo propor um meio de utilizar a tecnologia Blockchain no processo de trabalho em tecnologia BIM.

\subsection{Fluxos de trabalho BIM}

A partir de trabalho pioneiro da PennState University, a CBIC (2016b) propôs fluxos de trabalho em tecnologia BIM adaptados às necessidades brasileiras. Neste artigo, empregam-se como modelo o fluxograma para as macrofases "projeto conceitual" e "anteprojeto". O fluxograma demonstra as diversas trocas de informações que venham a ocorrer entre os entes de projeto, que neste caso são o Arquiteto, o Engenheiro Estrutural e o Engenheiro de Instalações.

\subsection{Ethereum blockchain}

A plataforma Ethereum é uma implementação da tecnologia Blockchain, que segundo a Ethereum Foundation (2018), é uma plataforma descentralizada, a qual executa aplicações denominadas smart contracts (contratos inteligentes), sob a premissa de nunca haver interrupções, censura, ou interferências de terceiros. Para fins de desenvolvimento de aplicações, emprega-se a Ethereum Virtual Machine - EVM, que é capaz de executar códigos escritos na linguagem de programação Solidity. A execução de smart contracts é um processo também denominado transação. Para que as transações ocorram, o usuário deve efetuar o pagamento de uma taxa, que varia conforme a complexidade da operação a ser executada. A moeda internamente utilizada para o pagamento destas taxas é a criptomoeda Ether. Toda transação é validada por entes denominados mineradores, que por sua vez são recompensados pela validação das transações com Ether. 
O indivíduo que se propõe a realizar transações na Ethereum deve adquirir a criptomoeda Ether, utilizando o Real $(R \$)$, o Dólar (\$), ou qualquer outra moeda fiduciária. A Ether pode ser adquirida em sites chamados de Exchange ou de indivíduos, por um valor que dependerá das condições de mercado. Portanto, o custo de uma transação dependerá do preço da Ether e da quantia que será consumida na execução do smart contract (contrato inteligente).

\section{METODOLOGIA}

Esta pesquisa considerou um fluxo de trabalho em projetos hipotéticos de edificações elaborados mediante o emprego da tecnologia BIM. Após a realização de revisão bibliográfica, com enfoque em fontes brasileiras, foi estabelecida como delimitadora a proposta sugerida por CBIC (2016b, p. 72-73), que corresponde às macrofases "projeto conceitual" e "anteprojeto". O fluxo de trabalho escolhido foi empregado para a realização de simulações de ambiente de projeto em BIM com o uso de Blockchain para o registro de autorias.

Em seguida, foi realizado o levantamento de ferramentas de código-aberto para registro de autorias de documentos que utilizassem a Blockchain pública Ethereum. As ferramentas foram levantadas no repositório GitHub.com, através das palavras-chave "ethereum notary". O critério de apresentação de informações utilizado foi o de "melhor resposta".

Também foi uma etapa desta pesquisa a execução de testes extensivos sobre a ferramenta de código aberto escolhida. O objetivo desta etapa foi o entendimento do procedimento de registro de existência de arquivos em Blockchain, eventuais custos operacionais, e a busca de bugs (falhas de execução do software).

Por fim, demonstrou-se a viabilidade do emprego de tecnologia Blockchain integrada ao fluxo de trabalho em tecnologia BIM.

\section{RESULTADOS}

Durante as buscas no site GitHub, a palavra "ethereum" direcionou os resultados à Blockchain pública em que se propôs desenvolver o trabalho. Para refinar a busca, a palavra "notary" fez com que o buscador apresentasse resultados de ferramentas que serviam a função de registrar e verificar a validade de arquivos. Por exclusão, a aplicação ETH Notary foi escolhida, que é atribuída inicialmente a MOTA (2019). A ETH Notary é uma ferramenta que grava em Ethereum blockchain códigos hash.

O código hash é o resultado de um processo chamado hashing onde um algoritmo analisa um arquivo digital e produz uma sequência de números e letras, chamado de código hash. O processo de hashing é unidirecional, o que a impede que a partir de um código hash seja produzida uma cópia do arquivo que o originou. Código hash produzido por um algoritmo específico sempre será o igual para um mesmo arquivo. Assim, alterações internas no arquivo implicariam em um novo hash, ao passo que a modificação do nome do arquivo não implicaria na produção de um novo código hash por não alterar a estrutura do arquivo.

Para o registro de um arquivo de projeto em uma rede Blockchain foi constatado que não é necessária sua transferência (upload) para a rede Ethereum blockchain. Conforme esquematizado na Figura 1, deve-se submeter o arquivo a ser validado à leitura do algoritmo Keccak-256, gerador de código hash. O usuário que comanda a tarefa possui uma identidade que será atrelada ao registro da identidade do arquivo. Por fim, é executado o smart contract (Figura 1).

\subsection{Emprego de Blockchain em tecnologia BIM}

Durante as simulações, o emprego da ferramenta ETH Notary em tecnologia BIM ocorreu na etapa de envio de arquivos de referência. Assim, hipoteticamente, se um projeto arquitetônico conceitual tinha que ser transmitido para a elaboração do projeto conceitual de estruturas sua identidade era registrada em uma rede blockchain. Analogamente, o projeto conceitual de estruturas foi registrado em Blockchain antes de ser disponibilizado para as demais disciplinas, mesmo se fosse o caso de ser utilizado como referência a uma nova versão do projeto arquitetônico conceitual. Para fins de reclamação de autoria ou cobrança de responsabilidade técnica, foi mantido o controle das dependências de cada projeto elaborado, conforme demonstrado no Quadro 1. 
Figura 1: Fluxograma de registro de documentos em Ethereum Blockchain com emprego de ETH Notary.

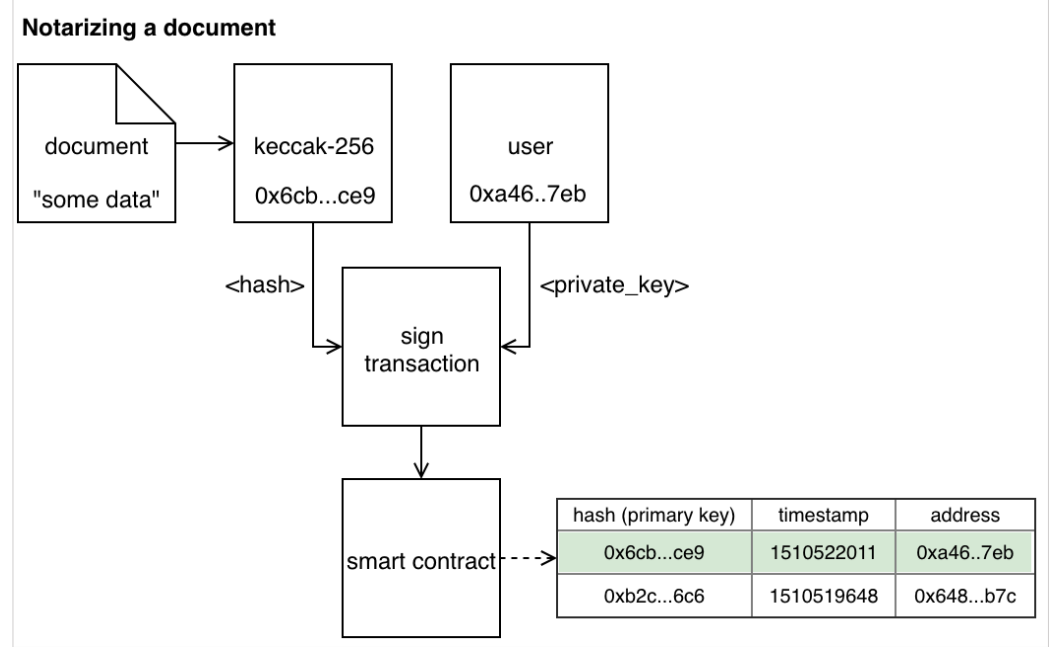

Fonte: Adaptado de MOTA (2019).

Quadro 1: Controle de dependências de projetos

\begin{tabular}{|c|c|c|c|c|c|}
\hline DISCIPLINA/PROJETO & $\begin{array}{c}\text { CÓDIGO } \\
\text { HASH }\end{array}$ & $\begin{array}{c}\text { DISCIPLINA/PROJETO DE } \\
\text { REFERÊNCIA - A }\end{array}$ & $\begin{array}{c}\text { CÓDIGO } \\
\text { HASH }\end{array}$ & $\begin{array}{c}\text { DISCIPLINA/PROJETO DE } \\
\text { REFERÊNCIA - B }\end{array}$ & $\begin{array}{c}\text { CÓDIGO } \\
\text { HASH }\end{array}$ \\
\hline $\begin{array}{c}\text { ARQUITETURA.FASE_DE_PR } \\
\text { OJETO_CONCEITUAL- } \\
\text { VERSAO_1.IFC }\end{array}$ & $\begin{array}{c}\text { 0x0dcf...20 } \\
5 \mathrm{~b} 41 \mathrm{a} 822\end{array}$ & - & - & - \\
\hline $\begin{array}{c}\text { ESTRUTURAL.FASE_DE_PR } \\
\text { OJETO_CONCEITUAL- } \\
\text { VERSAO_1.IFC }\end{array}$ & $\begin{array}{c}\text { 0x400ac4... } \\
52063\end{array}$ & $\begin{array}{c}\text { ARQUITETURA.FASE_DE_PR } \\
\text { OJETO_CONCEITUAL- } \\
\text { VERSAO_1.IFC }\end{array}$ & $\begin{array}{c}\text { 0x0dcf...20 } \\
5 b 41 \mathrm{a} 822\end{array}$ & & - \\
\hline & & $\begin{array}{c}\text { ARQUITETURA.FASE_DE_PR } \\
\text { OJETO_CONCEITUAL- } \\
\text { VERSAO_1.IFC }\end{array}$ & $\begin{array}{c}\text { 0x0dcf...20 } \\
\text { 5b41a822 }\end{array}$ & $\begin{array}{c}\text { ESTRUTURAL.FASE_DE_ DROJETO_CONCEITUAL- } \\
\text { VRERSAO_1.IFC }\end{array}$ & $\begin{array}{c}\text { 0x400ac } \\
4 . . .5206 \\
3\end{array}$ \\
\hline
\end{tabular}

Fonte: Os autores.

\section{DISCUSSÃo}

A dificuldade em se atribuir responsabilidades, ou de reconhecimento autoral de trabalhos em ambiente BIM, pode ser superada com o emprego da tecnologia Blockchain, que possui dispositivos, como o registro distribuído e a descentralização, que a tornam imutável. Outro aspecto observado é que a tecnologia Blockchain não é um banco de dados destinado ao armazenamento de arquivos de projeto, mas sim destinado ao armazenamento do estado de arquivos.

A aplicação ETH Notary permite que os entes de projeto registrem e consultem a autoria de projetos em Ethereum Blockchain. Assim, como foi demonstrado, a equipe de projeto deve manter um controle das dependências de projetos, que relacione os arquivos BIM ao código hash gerado pelo algoritmo.

Em um fluxo de projeto em tecnologia BIM, as trocas de arquivos de referências entre os entes de projeto ocorrem quantas vezes forem necessárias (CBIC 2016b, p. 72-73). Conforme foi apresentado, todo arquivo de referência deve ter seu estado gravado em Blockchain. Contudo, a execução do smart contract (contrato inteligente) é um evento dependente do pagamento de uma taxa, em Ether. Este custo da execução de um smart contract representa um aspecto negativo da tecnologia, pois o preço da criptomoeda Ether pode variar de acordo com fatores ligados ao balanço entre a oferta e a demanda desta criptomoeda. Por outro lado, tendo em vista a disseminação do uso da tecnologia BIM na construção civil, a qual subentende o aumento da demanda pelo controle de autoria dos projetos, a relação custo benefício é positiva.

\section{REFERÊNCIAS}

CBIC. Coletânea Implementação do BIM para Construtoras e Incorporadoras: Parte 5 - Formas de contratação BIM. Brasília, DF: Câmara Brasileira da Indústria da Construção, 2016a. v. 5

CBIC. Coletânea Implementação do BIM para Construtoras e Incorporadoras: Parte 4 - Fluxos de trabalho BIM. Brasília, DF: Câmara Brasileira da Indústria da Construção, 2016b. v. 4 
ETHEREUM FOUNDATION. Ethereum Project. Disponível em: < https://ethereum.org/>. Acesso em: 7 dez. 2018.

FOSTER, L. L. Legal Issues and Risks Associated with Building Information Modeling Technology. 2008. University of Kansas, 2008. Disponível em: <https://kuscholarworks.ku.edu/handle/1808/4264>. Acesso em: 22 ago. 2018.

MCADAM, B. Building information modelling: the UK legal context. International Journal of Law in the Built Environment, v. 2, n. 3, 2010, p. 246-259.

MOTA, M. ETHNnotary. Disponível em: <https://github.com/miguelmota/ethnotary>. Acesso em: 28 jan. 2019.

SACKS, R. et al. BIM Handbook: a guide to building information modeling for owners, designers, engineers, contractors, and facility managers. 3a. ed. Hoboken, New Jersey: Wiley, 2018, 659 p.

SCHAPKE, S.-E. et al. Collaborative Data Management. In: Building Information Modeling. Cham: Springer International Publishing, 2018. p. 251-277.

STOUGIANNOS, L.; MAGNERON, A. BIM, Blockchain and the Smart Construction Contract - Lexology. Disponível em: <https://www.lexology.com/library/detail.aspx?g=a94913aa-9135-4547-a5e4-5a114dc3d502>. Acesso em: 30 maio. 2018.

UDOM, K. BIM: mapping out the legal issues | NBS I 2012, february. Disponível em:

<https://www.thenbs.com/knowledge/bim-mapping-out-the-legal-issues>. Acesso em: 22 ago. 2018.

ZHENG, Z. et al. An Overview of Blockchain Technology: Architecture, Consensus, and Future Trends. In: 2017 IEEE International Congress on Big Data (BigData Congress), Anais...2017. 